\title{
DISTRIBUIÇÃO DAS ESPÉCIES DE CHAETOGNATHA NA PLATAFORMA CONTINENTAL DE SERGIPE E ALAGOAS
}

\author{
ARAÚJO, H.M.P. \& RIBEIRO, V.A.* \\ Núcleo de Ecossistemas Costeiros, Departamento de Biologia, Universidade Federal de Sergipe \\ haraujo@ufs.br
}

\begin{abstract}
Araújo, H.M.P. \& Ribeiro, V.A. 2005. Chaetognatha species distribution in the continental shelf off Sergipe and Alagoas States. Braz. J. Aquat. Sci. Technol. 9(1):19-23. ISSN 1808-7035. The present study investigated the Chaetognatha species distribution in the continental shelf off Sergipe and Alagoas States and its relation to the abiotic variables. Samples were collected at eighteen stations along six transects, perpendicular to the coast line in December (2001 and 2002) and June (2002 and 2003), corresponding to the dry and rainy seasons, respectively. Oblique hauls were done with plankton net, mesh size $200 \mu \mathrm{m}$. Temperature, salinity, $\mathrm{pH}$, dissolved oxygen and nutrients were measured in three levels of the water column. Subsamples were taken using a Stempel pippette. Correlations analysis between Chaetognatha distribution and environmental parameters were studied using canonical correspondence analysis. Sagitta tenuis and $S$. enflata were the most adundant species, following by $S$. hispida, Sagitta spp., Krohnitta pacifica, Pterosagitta draco and S. bipunctata. Density values ranged between 9 ind. $\mathrm{m}^{-3}$, in December 2002, and 4,473 ind. $\mathrm{m}^{-3}$ in June 2002, when Sagitta tenuis and S. enflata were more abundant. Density average values decreased from stations located at 10 meters to that located at 30 meters depth. Temperature, phosphate, salinity and water transparence were correlated $(p<0,05)$ to the Chaetognatha species distribution.
\end{abstract}

Keywords: Chaetognatha; Shelf; Distribution; Sergipe; Alagoas

\section{INTRODUÇÃO}

O Filo Chaetognatha tem sido estudado sobre vários aspectos e nas diversas regiões do Brasil. Os primeiros trabalhos foram realizados na região norte por Vanucci \& Hosoe (1952) e Hosoe (1956). Ainda nesta região Sousa \& Lopes (1998) citam a ocorrência de 10 espécies. Almeida Prado (1960; 1961a; b; 1968) e Liang \& Vega-Pérez (1994) estudaram aspectos ecológicos das espécies registradas no sudeste do Brasil. Resgalla \& Montú (1995) abordaram a distribuição espacial e temporal de dezoito espécies para a costa sul brasileira.

Em águas costeiras e oceânicas do nordeste brasileiro este Filo tem sido freqüentemente estudado juntamente com os demais grupos zooplanctônicos (Gusmão, 1986 e 2000; Nascimento-Vieira et al., 1990 e Neumann-Leitão et al., 1998 e 1999).

O presente trabalho teve como objetivos estudar a composição e a distribuição das espécies de Chaetognatha na plataforma continental dos Estados de Sergipe e Alagoas.

${ }^{*}$ Bolsista PIBIC/CNPq

\section{MATERIAL E MÉTODOS}

As amostras de plâncton foram coletadas durante as campanhas oceanográficas realizadas em dezembro de 2001 e 2002 e junho de 2002 e 2003, correspondendo, respectivamente, aos períodos seco e chuvoso da região, em 18 estações, distribuídas em isóbatas de 10, 20 e 30 metros (Figura 1).

Os arrastos foram oblíquos, realizados com rede cônica-cilíndrica, com $200 \mu \mathrm{m}$ de abertura de malha, equipada com fluxômetro. As amostras foram fixadas em formalina a $4 \%$ e neutralizadas com bórax.

Para determinação das variáveis abióticas (salinidade, pH, oxigênio dissolvido, amônia, nitrito, nitrato, fosfato e silicato) as amostras de água foram coletadas com garrafa de Van Dorn, nos três níveis da coluna d'água (superfície, meio e fundo) e as análises dos parâmetros químicos foram realizadas pelo Laboratório de Química Ambiental da UFS.

$\mathrm{Na}$ análise das amostras de zooplâncton foi utilizado o método da subamostragem com pipeta de Stempel de $5 \mathrm{~mL}$.

Para testar a variação sazonal foi utilizado o teste ANOVA e o de comparações múltiplas de TukeyKramer, com um nível de significância de $95 \%$. Os cálculos foram realizados por meio do programa computacional STATISTICA 5.1. A integração dos da- 
dos bióticos e abióticos foi avaliada através da análise canônica de correspondência (Ter Braak, 1987; 1989).

\section{RESULTADOS E DISCUSSÃO}

Durante o período estudado seis espécies do Filo Chaetognatha foram identificadas: Sagitta tenuis, $S$. enflata, S. hispida, S. bipunctata, Krohnitta pacifica e Pterosagitta draco.

Os valores totais de densidade registrados foram de 22.155 ind. $\mathrm{m}^{-3}$ no período chuvoso e 4.984 ind. $\mathrm{m}^{-3}$ no período de estiagem (Tabela 1 ). A densidade decresceu da isóbata de 10, com valor total de 13.702 ind. $\mathrm{m}^{-3}$ para a de 30 metros com 3.466 ind. $\mathrm{m}^{-3}$ (Tabela 2).

Sagitta tenuis foi a espécie mais freqüente e abundante nos períodos amostrados, com valor total de densidade de 18.234 ind. $\mathrm{m}^{-3}$, no período chuvoso e de 3.707 ind. $\mathrm{m}^{-3}$ no período de estiagem. Além da variação temporal, essa espécie apresentou valor total de 11.212 ind. $\mathrm{m}^{-3} \mathrm{em}$ isóbata de 10 metros, decrescendo nas isóbatas de 20 metros ( 8.281 ind. $\mathrm{m}^{-3}$ ) e 30 metros (2.447 ind. $\mathrm{m}^{-3}$ ). De acordo com Resgalla \& Montú (1995), S. tenuis é uma espécie epiplanctônica, amplamente distribuída, e seus registros na costa sul do Brasil, coincidiram com a presença da Água Costeira.

Sagitta enflata foi a segunda espécie mais abundante e freqüente, com valor total de densidade de 3.207 ind. $\mathrm{m}^{-3}$ no período chuvoso e 908 ind. $\mathrm{m}^{-3}$ no período de estiagem. A espécie apresentou variação espacial, com valor total de 2.107 ind. $\mathrm{m}^{-3}$, em isóbata de 10 metros e de 870 ind. $\mathrm{m}^{-3} \mathrm{em}$ isóbata de 30 metros.

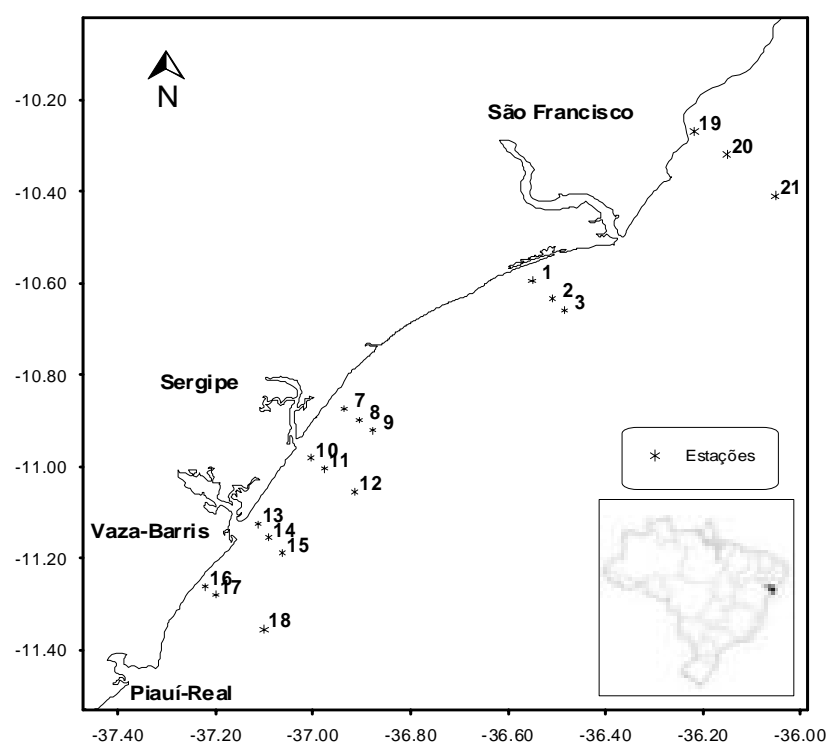

Figura 1 - Plataforma continental de Sergipe e Alagoas. Localização das estações de coleta.
Na região sul e sudeste do país, $S$. enflata foi a espécie mais freqüente e abundante, principalmente, em águas superficiais da plataforma e da zona oceânica (Almeida Prado, 1961b). Esses resultados foram confirmados por Resgalla \& Montú (1995) para o sul do Brasil. No Nordeste, Gusmão (1986) e Neumann-Leitão et al. (1999) citam esta espécie como a mais comum em águas costeiras e oceânicas.

Sagitta hispida foi mais abundante no período chuvoso $\left(474\right.$ ind $\left.\mathrm{m}^{-3}\right)$. O valor total de densidade dessa espécie foi maior na isóbata de 20 metros (263 ind $\left.\mathrm{m}^{-3}\right)$. S. hispida tem sido registrada na plataforma continental do nordeste do Brasil (Gusmão, 2000), do sudeste (Almeida Prado, 1961a; b; 1968; Liang \& VegaPérez, 1994; Marazzo et al., 1997) e do sul (Resgalla \& Montú,1995).

Sagitta bipunctata foi pouco freqüente, sendo registrada somente no mês de dezembro de 2002 na isóbata de 20 metros. Tem ampla distribuição na costa brasileira com ocorrência na região norte (Vannucci \& Hosoe, 1952; Sousa \& Lopes, 1998), nordeste (Neumann-Leitão et al., 1999; Gusmão, 2000), sudeste (Almeida Prado, 1961a) e sul do Brasil (Resgalla \& Montú, 1995).

Pterosagitta draco foi pouco freqüente e abundante, registrada somente no mês de junho de 2003 , na isóbata de 30 metros. $P$. draco é epipelágica com distribuição em regiões tropicais e subtropicais (Boltovskoy, 1999). Almeida Prado (1961a) considerou esta espécie oceânica de águas quentes e de alta salinidade. Na costa do Rio Grande do Sul, Resgalla \& Montú (1995) registraram esta espécie na plataforma externa e talude continental.

Krohnitta pacifica foi mais abundante durante 0 período de estiagem (161 ind. $\mathrm{m}^{-3}$ ), quando os valores de temperatura da água foram mais baixos. Hosoe (1956), Almeida-Prado (1961a) e Resgalla \& Montú (1995) registraram esta espécie na costa brasileira nas regiões de plataforma e oceânica.

Comparando-se os dados das diferentes isóbatas durante o período estudado, verificou-se, através do teste ANOVA e de comparações múltiplas de TukeyKramer, que o efeito da sazonalidade foi marcante somente na isóbata de 30 metros ( $p=0,0001)$, o que pode indicar influência de diferentes massas de águas oceânicas nessa região. Nas demais isóbatas a variação sazonal não é estatisticamente significativa (Figura 2).

A análise canônica de correspondência revelou que das variáveis ambientais analisadas, seis apresentaram relação significativa $(p<0,05)$ com os dados dados bióticos: transparência da água ( $\mathrm{Tr}$ ), temperatura da camada superficial (Ts) e intermediária (Tm) da coluna d'água, salinidade da camada intermediária $(\mathrm{Sm})$, fosfato de superfície $\left(\mathrm{PO}_{4} \mathrm{~s}\right)$ e fundo $\left(\mathrm{PO}_{4} \mathrm{f}\right)$. 
Braz. J. Aquat. Sci. Technol., 2005, 9(1):19-23.

Tabela 1 - Plataforma continental dos Estados de Sergipe e Alagoas. Dados de densidade total (T), densidade media (média) e desvio padrão (DP) das espécies de Chaetognatha em dezembro(2001 e 2002 e junho (2002 e 2003).

\begin{tabular}{l|rrr|rrr|rrr}
\hline & \multicolumn{3}{|c|}{ Dez/01 } & \multicolumn{3}{c|}{ Dez/02 } & \multicolumn{3}{c}{ Total Geral } \\
\hline Espécies & \multicolumn{1}{|c|}{ T } & Média & \multicolumn{1}{c|}{ DP } & \multicolumn{1}{c}{ T } & Média & \multicolumn{1}{c}{ DP } & \multicolumn{1}{c}{ T } & Média & DP \\
\hline Pterosagitta draco (Ptdr) & 0,0 & 0,0 & 0,0 & 0,0 & 0,0 & 0,0 & 0,0 & 0,0 & 0,0 \\
Sagitta bipunctata (Sabi) & 0,0 & 0,0 & 0,0 & 1,0 & 0,1 & 0,2 & 1,0 & 0,0 & 0,2 \\
Sagitta enflata (Saen) & 467,2 & 26,0 & 26,2 & 440,6 & 24,5 & 30,2 & 907,8 & 25,2 & 27,9 \\
Sagitta hispida (Sahi) & 19,0 & 1,1 & 2,5 & 0,0 & 0,0 & 0,0 & 19,0 & 0,5 & 1,8 \\
Sagitta spp (Sagi) & 173,5 & 9,6 & 18,0 & 14,7 & 0,8 & 2,1 & 188,1 & 5,2 & 13,4 \\
Sagitta tenuis (Sate) & 2595,4 & 144,2 & 156,5 & 1111,6 & 61,8 & 74,6 & 3707,0 & 103,0 & 127,9 \\
Krohnitta pacifica (Krpa) & 103,7 & 5,8 & 6,7 & 56,8 & 3,2 & 12,4 & 160,5 & 4,5 & 9,9 \\
\hline Total & $\mathbf{3 3 5 8 , 8}$ & $\mathbf{1 8 6 , 6}$ & $\mathbf{1 6 0 , 6}$ & $\mathbf{1 6 2 4 , 7}$ & $\mathbf{9 0 , 3}$ & $\mathbf{1 0 9 , 1}$ & $\mathbf{4 9 8 3 , 5}$ & $\mathbf{1 5 7 , 8}$ & $\mathbf{1 4 3 , 9}$ \\
\hline
\end{tabular}

\begin{tabular}{|c|c|c|c|c|c|c|c|c|c|}
\hline \multirow[b]{2}{*}{ Espécies } & \multicolumn{3}{|c|}{ Jun/02 } & \multicolumn{3}{|c|}{ Jun/03 } & \multicolumn{3}{|c|}{ Total Geral } \\
\hline & $T$ & Média & DP & $\mathbf{T}$ & Média & DP & $T$ & Média & DP \\
\hline Pterosagitta draco (Ptdr) & 0,0 & 0,0 & 0,0 & 5,3 & 0,3 & \begin{tabular}{l|l}
1,3 \\
\end{tabular} & 5,3 & 0,1 & 0,9 \\
\hline Sagitta bipunctata (Sabi) & 0,0 & 0,0 & 0,0 & 0,0 & 0,0 & 0,0 & 0,0 & 0,0 & 0,0 \\
\hline Sagitta enflata (Saen) & 2770,5 & 153,9 & 177,0 & 437,2 & 24,3 & 26,6 & 3207,7 & 89,1 & 141,0 \\
\hline Sagitta hispida (Sahi) & 0,0 & 0,0 & 0,0 & 474,0 & 26,3 & 33,2 & 474,0 & 13,2 & 26,7 \\
\hline Sagitta spp (Sagi) & 0,0 & 0,0 & 0,0 & 138,4 & 7,7 & 8,8 & 138,4 & 3,8 & 7,3 \\
\hline Sagitta tenuis (Sate) & 17509,6 & 972,8 & 1067,5 & 723,2 & 40,2 & 42,1 & 18232,8 & 506,5 & 882,1 \\
\hline Krohnitta pacifica (Krpa) & 0,0 & 0,0 & 0,0 & 97,2 & 5,4 & 6,2 & 97,2 & 2,7 & 5,1 \\
\hline Total & 20280,1 & 1126,7 & 1114,2 & 1875,3 & 104,2 & 62,4 & 22155,4 & 615,4 & 934,7 \\
\hline
\end{tabular}

Tabela 2 - Plataforma continental dos Estados de Sergipe e Alagoas. Total (T), Média e Desvio Padrão (DP) das espécies de Chaetognatha nas isóbatas de $10 \mathrm{~m}, 20 \mathrm{~m}$ e $30 \mathrm{~m}$, durante o período estudado.

\begin{tabular}{|c|c|c|c|c|c|c|c|c|c|}
\hline \multirow[b]{2}{*}{ Espécies } & \multicolumn{3}{|c|}{ Isóbata de $10 \mathrm{~m}$} & \multicolumn{3}{|c|}{ Isóbata de $20 \mathrm{~m}$} & \multicolumn{3}{|c|}{ Isóbata de $30 \mathrm{~m}$} \\
\hline & $\mathbf{T}$ & Média & DP & Total & Média & DP & Total & Média & DP \\
\hline Pterosagitta draco & 0,00 & 0,00 & 0,00 & 0,00 & 0,00 & 0,00 & 5,35 & 0,22 & 1,07 \\
\hline Sagitta bipunctata & 0,00 & 0,00 & 0,00 & 0,96 & 0,04 & 0,19 & 0,00 & 0,00 & 0,00 \\
\hline Sagitta enflata & 2106,54 & 87,77 & 156,41 & 1138,49 & 47,44 & 75,51 & 870,44 & 36,27 & 39,58 \\
\hline Sagitta hispida & 195,29 & 8,14 & 20,97 & 262,96 & 10,96 & 25,50 & 34,77 & 1,45 & 5,44 \\
\hline Sagitta spp & 64,39 & 2,68 & 5,82 & 196,47 & 8,19 & 15,51 & 65,67 & 2,74 & 6,80 \\
\hline Sagitta tenuis & 11212,19 & 467,17 & 636,35 & 8281,11 & 345,05 & 877,77 & 2446,53 & 101,94 & 189,73 \\
\hline Krohnitta pacifica & 124,08 & 5,17 & 11,66 & 90,87 & 3,79 & 5,91 & 42,77 & 1,78 & 2,57 \\
\hline Total & 13702,5 & 570,9 & 726,4 & 9970,9 & 415,5 & 898,3 & 3465,5 & 144,4 & 217,5 \\
\hline
\end{tabular}

Na Figura 3 estão plotadas as espécies e as principais variáveis ambientais.

A temperatura e a salinidade da água foram as variáveis ambientais mais importantes na construção do primeiro eixo canônico, com coeficientes de correlação de 0,63 e 0,57, respectivamente. As variáveis ambientais mais significativas na construção do segundo eixo canônico foram o fosfato do fundo e do meio da coluna d'água, com coeficientes de correlação de 0,41 e 0,29, respectivamente. Os parâmetros ambientais medidos explicaram $40 \%$ das variações dos dados bióticos.

A plataforma continental de Sergipe é uma das regiões marinhas mais estreitas do Brasil, com talude extremamente abrupto e é recortada por dois grandes canyons (São Francisco e Japaratuba), que provavelmente, orientam a ressurgência de águas profundas (Água Central do Atlântico Sul - ACAS) mais frias e ricas em nutrientes, especialmente em fosfato, no período de estiagem. Entretanto, a massa d'água predominante na plataforma continental da região estudada é a Água Tropical, associada à Corrente do Brasil. A dinâmica sazonal das massas de água está também associada ao regime de chuvas e vazões dos rios (Paes, 2004).

$\mathrm{Na}$ porção rasa da plataforma continental de Sergipe e Alagoas a influência de massas de águas 


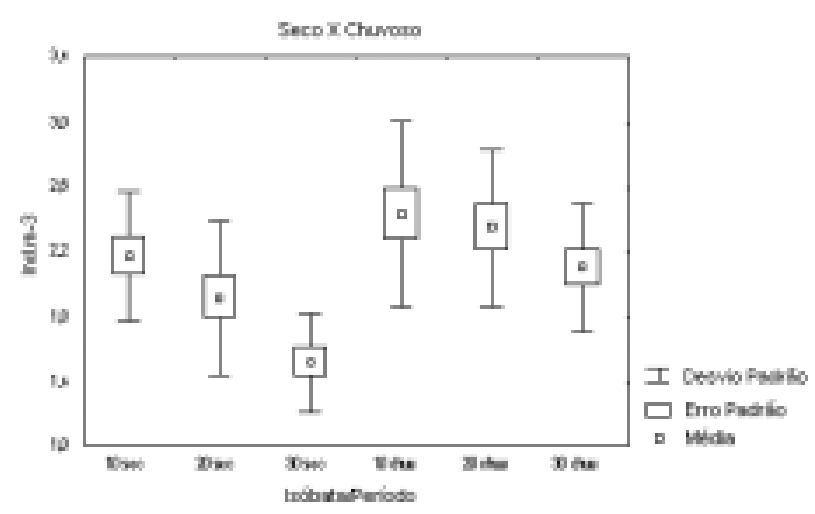

Figura 2 - Teste de comparações múltiplas de Tukey-Kramer, utilizando os dados de densidade (ind. $\mathrm{m}^{-3}$ ).

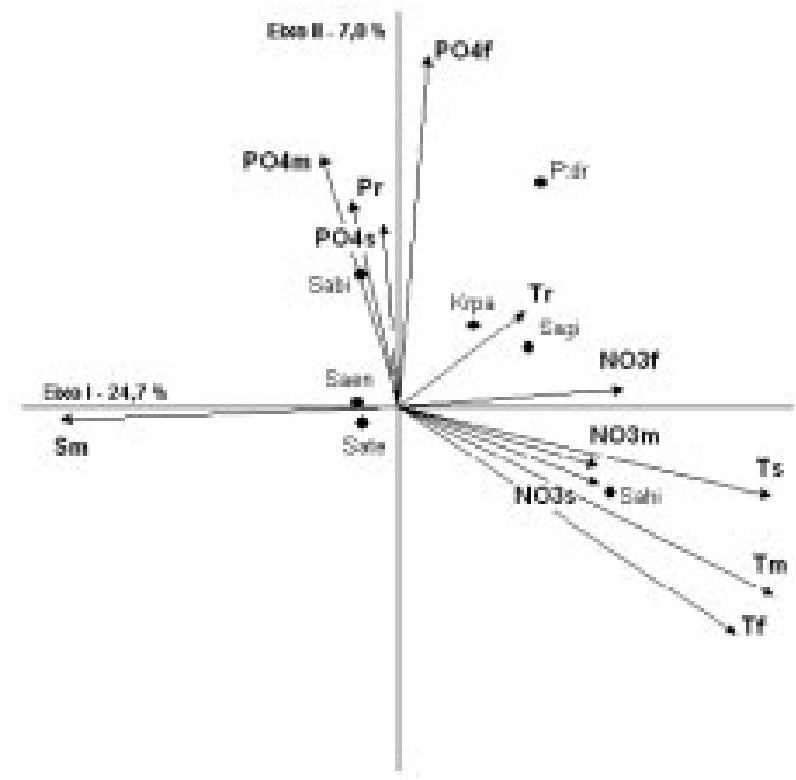

Figura 3 - Plataforma Continental do Estado de Sergipe e Alagoas. Análise canônica de correspondência, com projeção das espécies e das variáveis ambientais e percentuais de explicação de cada eixo.

oceânicas pode ser evidenciada pela ocorrência de Pterosagitta draco e Sagitta bipunctata, consideradas espécies oceânicas (Almeida Prado, 1961a). Pterosagitta draco foi registrada em estação de 30 metros, no período chuvoso, quando foram registrados valores de temperatura e nitrato mais elevados. Sagitta bipunctata foi registrada em estação de 20 metros, no período de estiagem, quando os valores de temperatura foram mais baixos.

Sagitta hispida também foi importante no período chuvoso. É uma espécie indicadora de águas tropicais e de plataforma (Almeida Prado, 1961a,b; Liang \& Vega-Pérez, 1994; Boltovskoy, 1999).

\section{CONCLUSÕES}

Seis espécies de Chaetognatha foram identificadas na plataforma continental de Sergipe e Alagoas: Sagitta tenuis, S. enflata, S. hispida, S. bipunctata, Pterosagitta draco e Krohnitta pacifica. Destas, $S$. tenuis e $S$. enflata foram as mais abundantes e freqüentes. Os valores de densidade foram mais elevados nas estações mais próximas da costa e em junho, mês inserido no período chuvoso da região.

\section{AGRADECIMENTOS}

À Petrobras/UN-SEAL pelo suporte financeiro para o desenvolvimento do projeto, ao CNPq pela bolsa de iniciação cientifica e ao Laboratório de Química Ambiental pela determinação dos parâmetros físicoquímicos.

\section{REFERÊNCIAS}

Almeida, M.S.P. 1960. A new species of Sagitta from the southern Brazilian coast. Bol. Inst. Oceonogr. São Paulo, 32(2):275-280.

Almeida, M.S.P. 1961a. Distribuição dos Chaetognatha no Atlântico Sul Ocidental. Bol. Inst. Oceonogr. São Paulo, 11:15-50.

Almeida Prado, M.S. 1961b. Chaetognatha encontrados em águas brasileiras. Bol. Inst. Oceonogr. São Paulo, 11:31-56.

Almeida Prado, M.S. 1968. Distribution and annual occurrence of Chaetognatha off Cananéia and Santos coast (São Paulo, Brazil). Bol. Inst. Oceonogr. São Paulo, 17:33-55.

Boltovskoy, D. 1999. South Atlantic zooplankton. Backhuys Publishers, Leidon. Vol.2, 869-1705p.

Gusmão, L.M.O. 1986. Chaetognatha planctônicos de províncias neríticas e oceânicas do Nordeste do Brasil. Dissertação (Mestrado). Universidade Federal de Pernambuco, Recife.

Gusmão, L.M.O. 2000. Comunidade zooplanctônica nas províncias nerítica e oceânica do Estado de Pernambuco - Brasil. Tese de doutorado. Universidade Federal de Pernambuco, Recife.

Hosoe, K. 1956. Chaetognatha from the Isle of Fernando de Noronha. Contrções Inst. Oceonogr., ser. Oceonogr. Biol., no 3, 9p.

Liang, T.H. \& Veja-Pérez, L.A. 1994. Studies on chaetognaths off Ubatuba region, Brazil. Distribution and abundance. Bol. Inst. Oceanogr. São Paulo, 42:73-84. 
Marazzo, A.; Machado, C.F. \& Nogueira, C.S.R. 1997. Notes on feeding of Chaetognatha in Guanabara Bay, Brazil. J. Plankton Res. 19:819-828.

Nascimento-Vieira, D.A.; Sant'Anna, E.M.E.; Luz, B. R.A. \& Neumann-Leitão, S. 1990. Zooplâncton nerítico e oceânico dos Estados de Alagoas e Pernambuco (Brasil). Trab. Oceonogr. Univ. Fed. PE. Recife, 21:81-101.

Neumann-Leitão, S.; Gusmão, L.A.O; NascimentoVieira, D.A., Silva, T.A.; Silva, A.P.; Porto Neto, F. F. \& Moura, M.C.O. 1998. Biodiversidade do mesozooplâncton no nordeste do Brasil. Trab. Oceonogr. Univ. Fed. PE, Recife, 216(1):27-34.

Neumann-Leitão, S.; Gusmão, L.A.O; Silva, T.A.; Nascimento-Vieira, D.A. \& Silva, A.P. 1999. Mesozooplankton biomass and diversity in coastal and oceanic waters off North-Eastern Brazil. Arch. Fish. Res. 47(2/3):153-165.

Paes, E.T. 2004. Análise integrada. In: Araujo, H.M.P. (coord.). Monitoramento da Plataforma Continental dos Estados de Sergipe e Alagoas sob influência de atividades petrolíferas. Relatório Técnico Final referente às quatro Campanhas Oceanográficas, apresentado à PETROBRAS em atendimento ao Contrato $\mathrm{N}^{\circ}$ 120.2.053.01-7 PETROBRAS/FAPESE e Convênio № 08/2001 FAPESE/UFS, Vol. 1, p: 718-734.

Resgalla, C. \& Montú, M. 1995. Quetognatos de la Plataforma Continental del Sur de Brasil. Inv. Mar. CICIMAR. México. 10(1-2):23-41.

Sousa, L.C.A. \& Lopes, M.J.S. 1998. Estudo dos Chaetognatha da região norte, área compreendida entre a foz do Rio Pará/PA à baia de São Marcos/ MA - Revizee ํㅜ1.

Ter Braak, C.J.F. 1987. The analysis of vegetationenvironment relationship by canonical correspondence analysis. Vegetation, 69:69-77.

Ter Braak, C.J.F. 1989. CANOCO - an extention of DECORANA to analyze species-environmental relationships. Hydrobiologia. 184:169-170.

Vanucci, M. \& Hosoe, K.1952. Resultados científicos do cruzeiro do "Baependi" e do "Vega" a llha da Trindade. Chaetognatha. Bol. Inst. Oceonogr. São Paulo, 3(1/2):5-30. 